\title{
Impact of AKAP6 polymorphisms on Glioma susceptibility and prognosis
}

\author{
Ming Zhang ${ }^{1}$, Yonglin Zhao ${ }^{2}$, Junjie Zhao ${ }^{3}$, Tingqin Huang ${ }^{1}$ and Yuan $\mathrm{Wu}^{4^{*}}$ (D)
}

\begin{abstract}
Purpose: Glioma is the most common primary malignant brain tumor with high mortality and poor prognosis. Our aim was to clarify the correlation between Kinase-anchored protein 6 (AKAP6) gene polymorphisms and glioma susceptibility and prognosis in Chinese Han population.

Methods: Five single-nucleotide polymorphisms (SNPs) of AKAP6 were genotyped by Agena MassARRAY in 575 glioma patients and 500 healthy controls. Logistic regression model was utilized to calculate odds ratios (OR) and 95\% confidence intervals (Cl). The associations between polymorphisms and survival were assessed using the logrank test, Kaplan-Meier analysis and Cox regression model.
\end{abstract}

Results: We found that rs2239647 polymorphism was strongly associated with an increased risk of glioma (OR $=1.90$, $p=0.007)$ and a worse prognosis for glioma, especially in high-grade glioma ( $H R=1.67, p=0.034)$. Stratified analysis showed that rs2239647 increased the risk of glioma in female $(\mathrm{OR}=1.62, p=0.016)$. Whereas, $\mathrm{rs} 4261436(\mathrm{HR}=0.70, p=$ $0.045)$ and $r s 17522122$ ( $H R=0.75, p=0.016)$ were associated with better prognosis of astrocytoma. In addition, we also found that surgical methods and chemotherapy are critical factors for the prognosis of glioma patients.

Conclusions: This study firstly provided evidence for the impact of AKAP6 polymorphisms on susceptibility and prognosis of glioma, suggesting AKAP6 variants might have potential roles in the etiology of glioma.

Keywords: Glioma, AKAP6, Single nucleotide polymorphisms, Susceptibility, Prognosis

\section{Introduction}

Glioma is a highly fatal disease that accounts for about $28 \%$ of all primary brain tumors in the United States. (1, 2). Gliomas are often fatal because many drugs that are effective against tumors throughout the body cannot cross the blood-brain barrier. Despite advances in treatment over the past few years, the prognosis for glioma patients remains poor, with a median overall survival rate (OS) of only 8 to 15 months $(3,4)$. The etiology of glioma involves various aspects, among which the role of genetic factors including genetic polymorphisms in the susceptibility and prognosis of glioma has aroused great concern. Single nucleotide polymorphisms of some genes have been shown to be associated with the risk or prognosis of glioma, such as Interleukin 4 Receptor (IL$4 R)$, EGF containing fibulin extracellular matrix protein 1 (EFEMP1), Regulator of telomere elongation helicase 1

\footnotetext{
* Correspondence: yiyi7229@163.com

${ }^{4}$ Department of Critical Care Medicine, the Second Affiliated Hospital of Xi'an Jiaotong University, \#157 Xiwu Road, Xi'an 710004, Shaanxi, China Full list of author information is available at the end of the article
}

(RTEL1), Cocaine and amphetamine regulated transcript (CART) and Isocitrate dehydrogenase 1 (IDH1) (5-9).

Kinase-anchored protein 6 (AKAP6), encoded by the AKAP6 gene, is a protein with diverse structures and is highly expressed in various brain regions and cardiac and skeletal muscle. AKAP6 is a member of the AKAP family proteins and performs important functions by binding to the regulatory subunit of protein kinase a (PKA) (10). PKA has been shown to be involved in many important signal transduction pathways. A previous study demonstrated effects of up-regulation of the cAMP/PKA pathway in glioblastoma cell (11). Genome-wide association studies (GWAS) have confirmed that the SNPs of AKAP6 were associated with brain-related diseases, such as Alzheimer's disease (12), anorexia nervosa (13), and poor cognitive, better memory abilities (14).

Based on previous results, we hypothesized that AKAP6 gene polymorphisms may be related to the pathogenesis of glioma. However, no literature supports the effect of AKAP6 polymorphisms on glioma. In this case-control

(c) The Author(s). 2019 Open Access This article is distributed under the terms of the Creative Commons Attribution 4.0 International License (http://creativecommons.org/licenses/by/4.0/), which permits unrestricted use, distribution, and reproduction in any medium, provided you give appropriate credit to the original author(s) and the source, provide a link to the Creative Commons license, and indicate if changes were made. The Creative Commons Public Domain Dedication waiver (http://creativecommons.org/publicdomain/zero/1.0/) applies to the data made available in this article, unless otherwise stated. 
study, we investigated the correlation between AKAP6 single nucleotide polymorphisms and glioma susceptibility and prognosis in the Han Chinese population.

\section{Methods}

\section{Study subjects}

In this study, 575 glioma patients (including 448 patients with astroglioma) and 500 healthy subjects were randomly recruited from Second Affiliated Hospital of Xi'an Jiaotong University. All patients were diagnosed with gliomas by imaging and histopathological, and all patients were unrelated. Demographic and clinical data were collected through standardized questionnaires and follow-up surveys, including age, sex, date of the first diagnosis, method of surgery, radiotherapy and/or chemotherapy program, date of last follow-up, and the condition of the patient (alive/dead) at the time of the last follow-up. Healthy subjects in the control group ruled out people with a history of cancer and people with a history of diseases associated with the brain and central nervous system. This study was approved by the ethics committee of Second Affiliated Hospital of Xi'an Jiaotong University and followed the Helsinki declaration. Each subject was informed of the purpose of our study and signed a written informed consent.

\section{DNA extracting and SNPs genotyping}

Genomic DNA was extracted from glioma patients' peripheral blood samples $(5 \mathrm{~mL})$ using Gold Mag-Mini DNA purification kit (Gold Mag Co. Ltd. Xian city, China). DNA concentrations were determined by the NanoDrop 2000 (Thermo Scientifc, Waltham, Massachusetts, USA). Multiplexed SNP Mass EXTENDED assay was designed by Agena MassARRAY Assay Design Software version 4.0 (Agena Co. Ltd., San Diego, CA, USA). SNP genotyping with a standard protocol was performed using Agena MassARRAY RS1000 (Agena Inc., San Diego, CA, USA). Agena Typer Software version 4.0 (Agena Inc., San Diego, California, USA) was used to management the data.

\section{Bioinformatics analysis}

Online software for HaploReg v4.1 (https://pubs.broadinstitute.org/ mammals/haploreg/haploreg.php) and SNPinfo Web Server (https://snpinfo. niehs.nih.gov/ snpinfo/index.html) were used to predict the possible functional effects on these SNPs.

\section{Statistical analysis}

The differences in demographic characteristics of study participants were evaluated using independent samples $\mathrm{T}$ test and Chi-square test. Deviation from Hardy-Weinberg equilibrium (HWE) was assessed using the Chi-square test. Odds ratio (OR) and 95\% confidence interval (CI) were calculated to estimate the relationships between SNPs and glioma risk using logistic regression analysis.
Multiple inheritance models (allele model, genotype model, dominant model, recessive model, and additive model) were assessed by PLINK software. Patient survival curves were plotted using the Kaplan-Meier method, and the log-rank test. Hazard ratio (HR) and 95\% confidence interval (CI) were calculated through univariate and multivariable Cox proportional hazard regression analysis to evaluate the effect of the AKAP6 genotypes on overall survival and progression-free survival (15-17). Statistical analysis was performed using SPSS Software version 20.0 (IBM, Armonk, New York, USA). A two-tailed $p<0.05$ was considered statistical significance.

\section{Results}

\section{Study subjects}

This study included 575 glioma patients and 500 agematched $(p=0.942)$ and gender-matched $(p=1.000)$ healthy controls, and the average ages were $40.53 \pm 13.90$ years and $40.45 \pm 18.08$ years respectively. The participants' demographic and clinical information was listed in Table 1, including age, gender, World Health Organization (WHO) grade and classification (18), surgical method, radiotherapy, chemotherapy and survival condition.

\section{Basic information of the selected SNPs}

Five SNPs in AKAP6 (rs1957021, rs2145587, rs2239647, rs4261436 and rs17522122) were genotyped. The basic information of selected SNPs and potential function predicted by HaploReg database about these variants were summarized in Additional file 4: Table S1. All SNPs conformed to the HWE equilibrium (all $p$ values were more than 0.05). The predicted results from the database showed that these SNPs might function as enhancer histone markers or by changing motifs.

\section{The SNPs of $A K A P 6$ and the risk of glioma}

Multiple inheritance models analysis (allele, genotype, dominant, recessive and additive) for the association between AKAP6 rs2239647 and risk of glioma are showed in Table 2. Our analysis revealed a relationship between AA genotype of rs2239647 and increased glioma risk in genotype model ( $\mathrm{OR}=1.88,95 \% \mathrm{CI}: 1.16-3.04, p=0.010)$ and recessive model (OR $=1.90,95 \% \mathrm{CI}: 1.19-3.03, p=0.007)$.

In addition, we conducted a stratified analysis to explore the effects of these SNPs on glioma susceptibility in a specific population. The significant results of stratified analysis are showed in Table 3 . The results showed that AA genotype at rs2239647 was significantly associated with increased glioma risk in populations over 40 years old (genotype model: $\mathrm{OR}=2.60, p=0.012$; recessive model: $\mathrm{OR}=2.83, p=0.006$ ) and in the male population (genotype model: $\mathrm{OR}=2.42, p=0.003$; recessive model: $\mathrm{OR}=2.49, p=0.009$ ). And, people with the rs2239647-AA genotype had a higher risk of astroglioma 
Table 1 Characteristics of glioma patients and healthy controls

\begin{tabular}{lllll}
\hline Characteristics & & $\begin{array}{l}\text { Cases } \\
(n=575)\end{array}$ & $\begin{array}{l}\text { Controls } \\
(n=500)\end{array}$ & $p$ \\
\hline Age, years & Mean \pm SD (year) & $40.53 \pm$ & $40.45 \pm$ & $0.942^{\mathrm{a}}$ \\
& & 13.90 & 18.08 & \\
$\leq 40$ & $279(49 \%)$ & $265(53 \%)$ & \\
& $>40$ & $296(51 \%)$ & $235(47 \%)$ &
\end{tabular}

Gender

\begin{tabular}{llll} 
& Male & $320(56 \%)$ & $279(56 \%)$ \\
& Female & $255(44 \%)$ & $221(44 \%)$ \\
WHO grade & I-II & $369(64 \%)$ \\
& III-IV & $206(36 \%)$ \\
astrocytoma & & $448(78 \%)$ \\
Surgical & STR \& NTR & $184(32 \%)$ \\
method & GTR & $394(68 \%)$ \\
Radiotherapy & Gamma knife & $365(63 \%)$ \\
& Conformal & $156(27 \%)$ \\
& radiotherapy & $57(10 \%)$ \\
Chemotherapy & Yes & $237(41 \%)$ \\
& No & $341(59 \%)$ \\
State of & Progress & $538(93 \%)$ \\
progress & No & $35(6 \%)$ \\
& Absent & $5(1 \%)$ \\
\hline
\end{tabular}

WHO World Health Organization, GTR Gross-total resection, NTR Near-total resection, STR Sub-total resection

a $p$ values was calculated by independent samples $T$ test

${ }^{\mathrm{b}} p$ values was calculated by Chi-square tests

than healthy controls (genotype model: $\mathrm{OR}=1.90, p=$ 0.012 ; recessive model: $\mathrm{OR}=1.92, p=0.009)$. Moreover, rs2145587 was associated with an increased risk of glioma in female (genotype model: $\mathrm{OR}=1.62, p=0.016$; dominant model: $\mathrm{OR}=1.57, p=0.017$ ).

\section{The SNPs of $A K A P 6$ and the prognostic of glioma}

The log-rank test was applied to analyze the associations between overall survival (OS) or progression free survival (PFS) and clinical factors, and the results indicated that gender, age, WHO grading, and radiotherapy factors were not related to the prognosis of patients $(p>0.05)$, while surgical methods and chemotherapy were significantly related to the prognosis of patients $(p<0.05)$ (Additional file 5: Table S2 and Additional file 1: Figure S1). We found that the prognosis of glioma patients undergoing total resection was better than patients who did not undergo complete resection (OS: $\log$-rank $p<0.001, \mathrm{HR}=$ $0.63, p<0.001$; PFS: log-rank $p<0.001$, HR $=0.59, p<$ $0.001)$. The prognosis of patients receiving chemotherapy was better than that of patients not receiving chemotherapy (OS: log-rank $p<0.001, \mathrm{HR}=0.67, p<0.001$; PFS: $\log$ $\operatorname{rank} p=0.012, \mathrm{HR}=0.81, p=0.025)$.
Table 2 Relationships between AKAP6 rs2239647 and glioblastoma risk

\begin{tabular}{|c|c|c|c|c|c|c|}
\hline \multirow[t]{2}{*}{ SNP ID } & \multirow[t]{2}{*}{ Model } & \multirow[t]{2}{*}{ Genotype } & \multirow[t]{2}{*}{ Case } & \multirow[t]{2}{*}{ Control } & \multicolumn{2}{|c|}{$\begin{array}{l}\text { Adjusted by age and } \\
\text { gender }\end{array}$} \\
\hline & & & & & OR $(95 \% \mathrm{Cl})$ & $p$ \\
\hline \multirow[t]{10}{*}{ rs2239647 } & \multirow[t]{3}{*}{ Allele } & $C$ & 817 & 746 & 1.00 & \multirow[t]{3}{*}{0.086} \\
\hline & & A & 329 & 254 & $\begin{array}{l}1.18 \\
(0.98-1.43)\end{array}$ & \\
\hline & & $\mathrm{CC}$ & 302 & 274 & 1.00 & \\
\hline & \multirow[t]{2}{*}{ Genotype } & $C A$ & 213 & 198 & $\begin{array}{l}0.98 \\
(0.76-1.26)\end{array}$ & 0.849 \\
\hline & & AA & 58 & 28 & $\begin{array}{l}1.88 \\
(1.16-3.04)\end{array}$ & 0.010 \\
\hline & \multirow[t]{2}{*}{ Dominant } & CC & 302 & 274 & 1.00 & \multirow[t]{2}{*}{0.494} \\
\hline & & CA-AA & 271 & 226 & $\begin{array}{l}1.09 \\
(0.85-1.38)\end{array}$ & \\
\hline & \multirow[t]{2}{*}{ Recessive } & $C C-C A$ & 515 & 472 & 1.00 & \multirow[t]{2}{*}{0.007} \\
\hline & & $\mathrm{AA}$ & 58 & 28 & $\begin{array}{l}1.90 \\
(1.19-3.03)\end{array}$ & \\
\hline & Additive & $\begin{array}{l}A A \text { vs } A B \\
\text { vs } B B\end{array}$ & - & - & $\begin{array}{l}1.18 \\
(0.97-1.42)\end{array}$ & 0.091 \\
\hline
\end{tabular}

SNP single nucleotide polymorphism, OR odds ratio, 95\% Cl 95\% confidence interval $p$ values were calculated by logistic regression analysis with adjustments for age and gender

Bold values indicate statistical significance $(p<0.05)$

We evaluated the effect of AKAP6 polymorphisms on the patient survival. Log-rank test and Kaplan-Meier analysis revealed the relationship between rs2239647 and OS and PFS in glioma patients (Table 4 and Fig. 1). We found that AKAP6-rs2239647 significantly affected the PFS of patients with high-level glioma (WHO grade III-IV), and patients with CA genotype had a better prognosis (PFS: log-rank $p=0.045, \mathrm{HR}=1.67, p=0.034$ ).

Subsequently, we analyzed the effect of AKAP6 polymorphisms on the prognosis of patients with astroglioma (Table 5 and Fig. 2). The results showed that AKAP6rs4261436 had a significant effect on the OS of patients, and patients with TC genotype had a poor prognosis (OS: $\log$-rank $p=0.038, \mathrm{HR}=0.70, p=0.045)$. AKAP6- rs175 22122 also had a significant effect on the OS of patients, and patients with TC genotype had a poor prognosis (OS: $\log$-rank $p=0.025, \mathrm{HR}=0.75, p=0.016$ ).

\section{Discussion}

This study confirmed the relationship between AKAP6 gene variation and glioma risk or prognosis in Han Chinese population. We found that AKAP6 single nucleotide polymorphisms rs2239647 and rs2145587 were associated with glioma susceptibility. More importantly, rs2239647, rs4261436 and rs17522122 were significantly correlated with the prognosis of patients. In addition, we also found that the extent of the surgical resection and 
Table 3 Stratified analysis of the relationships between AKAP6 polymorphisms and glioma risk

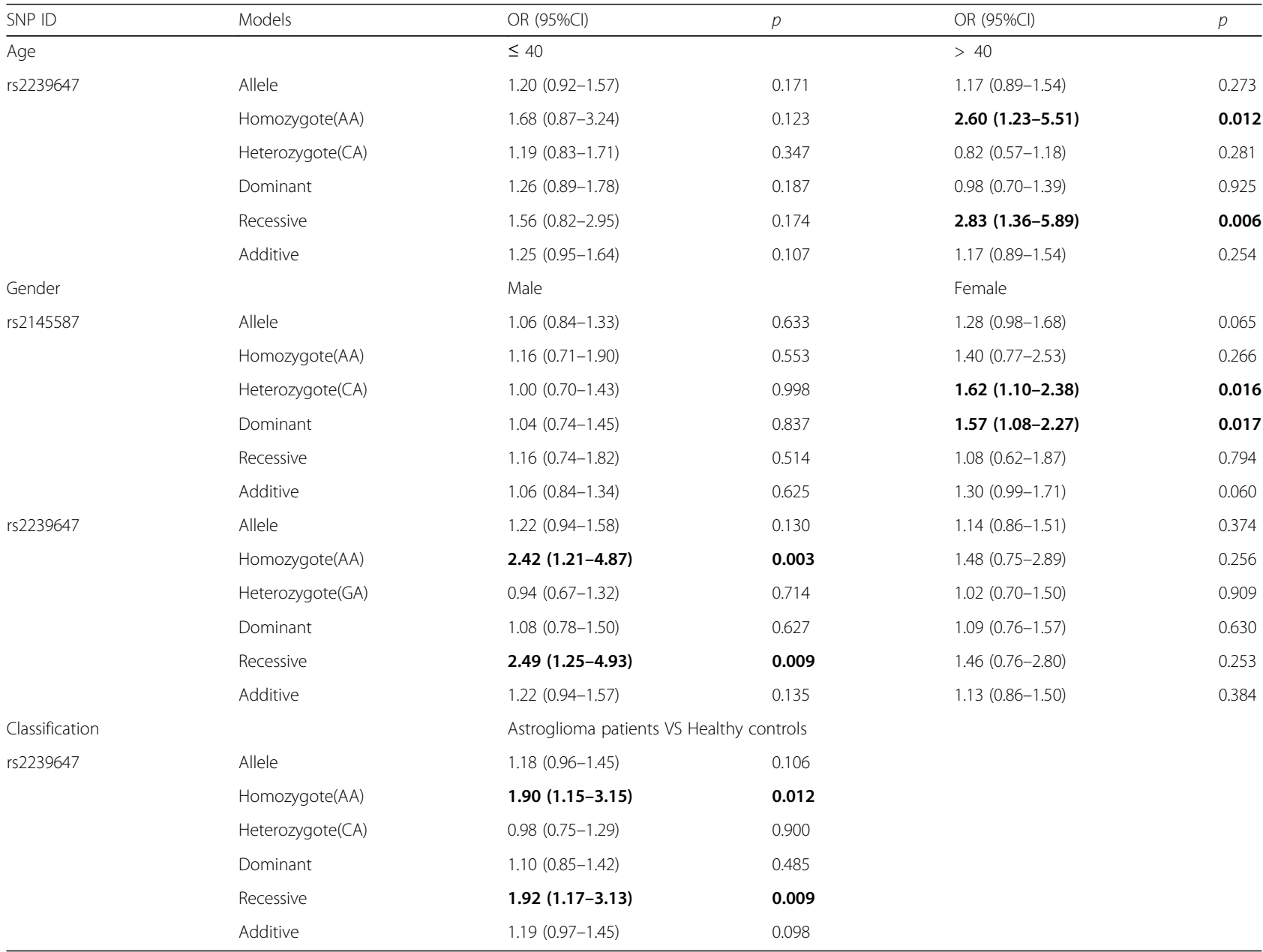

SNP single nucleotide polymorphism, OR odds ratio, 95\% Cl 95\% confidence interval

$p$ values were calculated by logistic regression analysis with adjustments for age and gender

Bold values indicate statistical significance $(p<0.05)$

Table 4 The association between rs2239647 and glioma patient OS and PFS

\begin{tabular}{|c|c|c|c|c|c|c|c|c|c|}
\hline \multirow[t]{2}{*}{ rs2239647 } & \multirow[t]{2}{*}{ Genotype } & \multicolumn{4}{|l|}{ OS } & \multicolumn{4}{|l|}{ PFS } \\
\hline & & Log-rank $p$ & SR (1-/3-year) & $\mathrm{HR}(95 \% \mathrm{Cl})$ & $p$ & Log-rank $p$ & SR (1-/3-year) & HR $(95 \% \mathrm{Cl})$ & $p$ \\
\hline & CC & 0.227 & $0.328 / 0.080$ & 1.00 & & 0.413 & $0.182 / 0.084$ & 1.00 & \\
\hline & CA & & $0.327 / 0.134$ & $1.15(0.86-1.54)$ & 0.346 & & $0.193 / 0.122$ & $1.13(0.84-1.51)$ & 0.424 \\
\hline & AA & & $0.224 / 0.034$ & $0.91(0.76-1.10)$ & 0.325 & & $0.138 / 0.039$ & $0.94(0.78-1.13)$ & 0.517 \\
\hline \multicolumn{10}{|c|}{ Low-grade glioma(I-II) } \\
\hline & CC & 0.124 & $0.324 / 0.077$ & 1.00 & & 0.267 & $0.159 /-$ & 1.00 & \\
\hline & CA & & $0.349 / 0.165$ & $0.96(0.66-1.39)$ & 0.818 & & $0.232 / 0.147$ & $0.93(0.64-1.35)$ & 0.686 \\
\hline & $\mathrm{AA}$ & & $0.270 /-$ & $0.80(0.64-1.01)$ & 0.065 & & $0.189 /-$ & $0.84(0.66-1.06)$ & 0145 \\
\hline \multicolumn{10}{|c|}{ High-grade glioma (III-IV) } \\
\hline & $\mathrm{CC}$ & 0.056 & $0.333 / 0.085$ & 1.00 & & 0.045 & $0.219 / 0.092$ & 1.00 & \\
\hline & CA & & $0.279 /-$ & $1.61(1.04-2.66)$ & 0.035 & & $0.106 /-$ & $1.67(1.04-2.67)$ & 0.034 \\
\hline & $\mathrm{AA}$ & & $0.143 /-$ & $1.19(0.87-1.63)$ & 0.268 & & $0.048 /-$ & $1.21(0.88-1.66)$ & 0.233 \\
\hline
\end{tabular}

OS Overall survival, PFS Progression free survival, SR Survival rate, HR Hazard ratio, 95\% Cl 95\% Confidence interval Log-rank $p$ values were calculated using the Chi-Square test

Bold values indicate statistical significance $(p<0.05)$ 


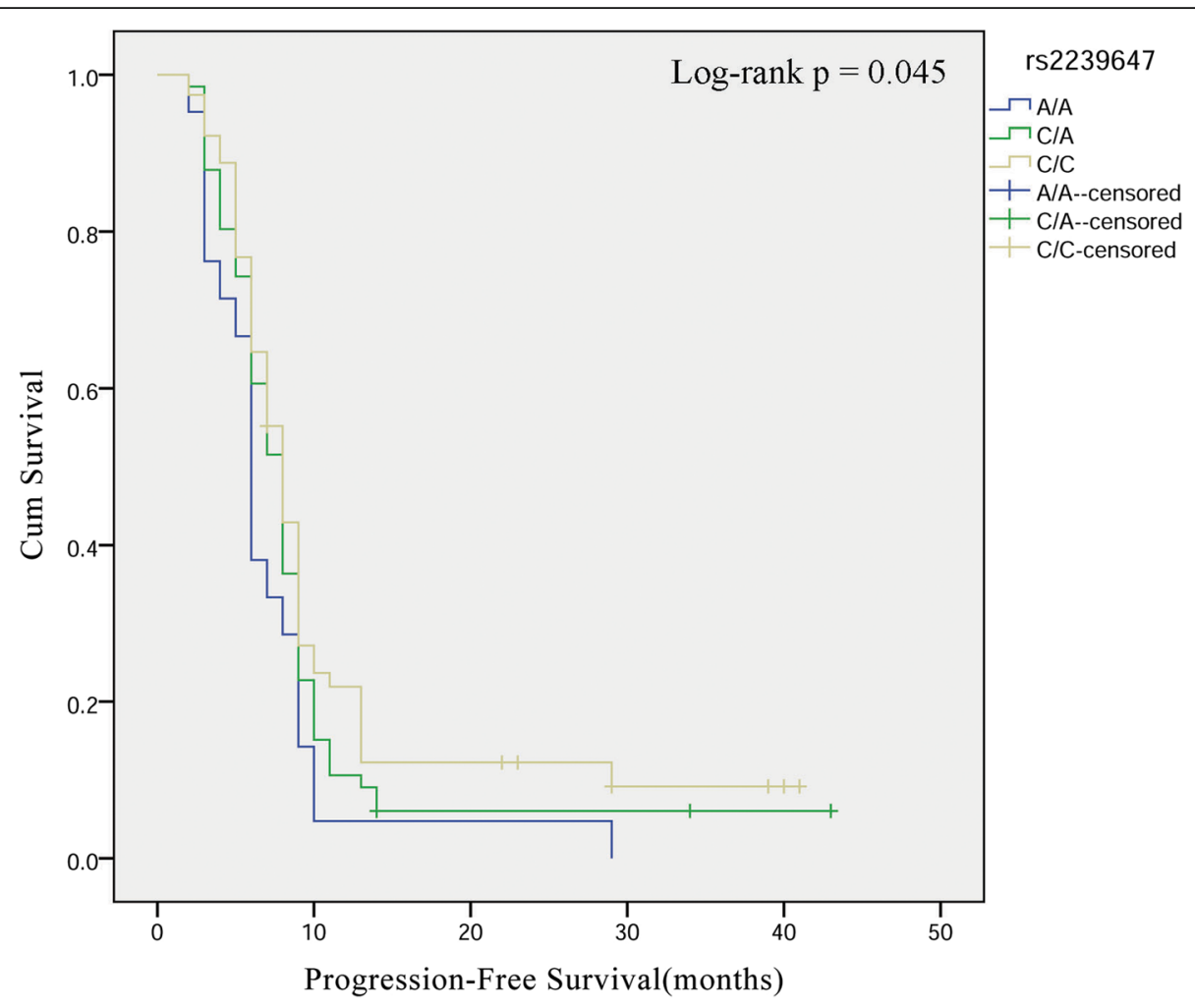

Fig. 1 Glioma patient survival based on AKAP6-rs2239647 polymorphism. Kaplan-Meier survival curves are plotted for and progression free survival.

chemotherapy are also key factors for the prognosis of glioma patients.

Previous study has found that AKAP6 protein is highly expressed in the brain (10), and we also used the GEPIA database (http://gepia.pku.cn/) to predict the expression of AKAP6 mRNA, the result showed that the mRNA level of AKAP6 gene was significantly up-regulated in glioma ( $p<0.05$, Additional file 2: Figure S2). This indicates that AKAP6 gene plays an important role in the occurrence and development of glioma.

SNPs in AKAP6 gene have been associated with several brain-related diseases/traits by GWAS. AKAP6- rs4296166 has been associated with risk of Alzheimer's disease (12), and rs2383378 was suggestively associated with anorexia nervosa (13). AKAP6-rs17522122 was associated with worse general fluid cognitive performance, verbal numerical reasoning and improved performance in reaction time and memory (14). In this study, it was found that the SNPs of AKAP6 were significantly correlated with the susceptibility and prognosis of glioma. At the same time, using GEPIA database analysis, we also found that AKAP6 gene also had a significant impact on the overall survival rate of low-grade glioma patients $(p<0.05$, Additional file 3: Figure S3). Combined with

Table 5 The association between rs4261436, rs17522122 and astrocytoma patient OS and PFS

\begin{tabular}{|c|c|c|c|c|c|c|c|c|c|}
\hline \multirow[t]{2}{*}{ SNP ID } & \multirow[t]{2}{*}{ Genotype } & \multicolumn{4}{|l|}{ OS } & \multicolumn{4}{|l|}{ PFS } \\
\hline & & Log-rank $p$ & SR (1-/3-year) & HR $(95 \% \mathrm{Cl})$ & $p$ & Log-rank $p$ & SR (1-/3-year) & HR $(95 \% \mathrm{Cl})$ & $p$ \\
\hline \multirow[t]{3}{*}{ rs4261436 } & $\pi$ & 0.038 & $0.258 / 0.033$ & 1.00 & & 0.176 & $0.152 /-$ & 1.00 & \\
\hline & $\mathrm{TC}$ & & $0.367 / 0.116$ & $0.70(0.49-0.99)$ & 0.045 & & $0.178 /-$ & $0.75(0.53-1.07)$ & 0.116 \\
\hline & CC & & $0.356 / 0.111$ & $0.81(0.64-1.02)$ & 0.078 & & $0.267 /-$ & $0.88(0.70-1.12)$ & 0.308 \\
\hline \multirow[t]{3}{*}{ rs17522122 } & GG & 0.025 & $0.268 / 0.049$ & 1.00 & & 0.053 & $0.137 /-$ & 1.00 & \\
\hline & GT & & $0.352 / 0.106$ & $0.75(0.59-0.95)$ & 0.016 & & $0.190 /-$ & $0.79(0.55-1.13)$ & 0.686 \\
\hline & $\Pi$ & & $0.341 / 0.042$ & $0.78(0.54-1.12)$ & 0.818 & & $0.268 /-$ & $0.78(0.62-0.99)$ & 0.038 \\
\hline
\end{tabular}

OS Overall survival, PFS Progression free survival, SR Survival rate, HR Hazard ratio, 95\% Cl 95\% Confidence interval Log-rank $p$ values were calculated using the Chi-Square test

Bold values indicate statistical significance $(p<0.05)$ 

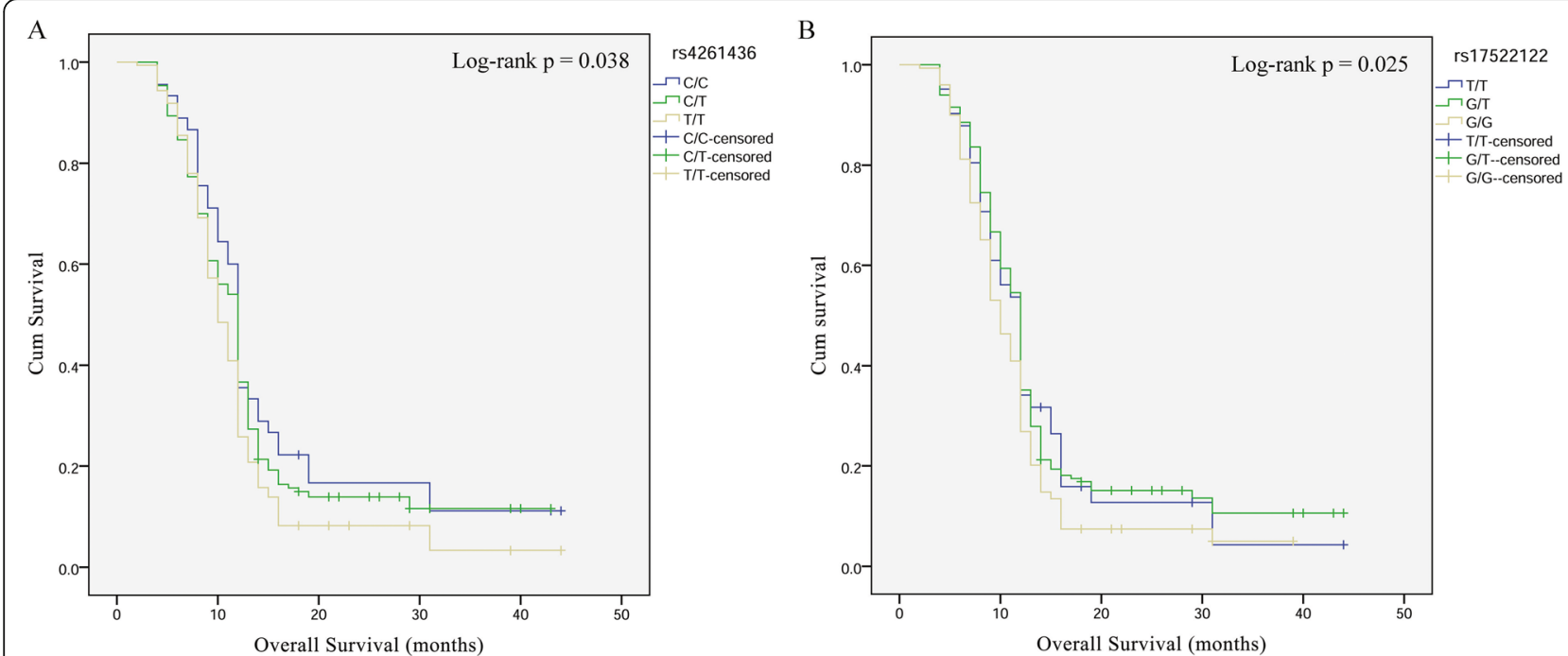

Fig. 2 Astroglioma patient survival based on AKAP6-rs4261436 (A) and -rs17522122 (B) polymorphisms. Kaplan-Meier survival curves are plotted for overall survival

the predicted function of SNPs, we hypothesized that the SNPs of APAK6 may affect gene expression and thereby affect the risk and prognosis of glioma.

PKA phosphorylation is central to the regulation of many cellular processes, and the specificity of PKA signaling is mediated in part by PKA binding to AKAPs. Previous studies have shown the role of cAMP/PKA pathway in glioblastoma cell lines and primary culture. By increasing the level of cAMP or activating PKA directly through cAMP analogues, the proliferation, differentiation and apoptosis of a-172 cells could be decreased (11). Therefore, the regulation of cAMP/PKA pathway may be a possible target for the treatment of malignant glioma. Although the function of AKAP6 gene in this process has not been studied in detail, its importance cannot be ignored. We will verify the function of AKAP6 gene in glioma through experiments in subsequent studies.

There are inevitably some limitations in this study. Firstly, there are regional limitations in sample selection. Subsequent sample selection should expand the geographical scope. Secondly, this study only carried out basic research, lack of functional verification experiments. In the following experiments, we will conduct experimental studies on gene expression of AKAP6 and function of SNPs. Despite the shortcomings, the results of this study provide a theoretical basis for the study of glioma susceptibility.

\section{Conclusion}

In summary, our results show that AKAP6 polymorphism is associated with the susceptibility and prognosis of glioma in the Chinese Han population. These associations may provide new directions for risk assessment of glioma and prognosis assessment of glioma patients.
However, our results need to be replicated in a larger sample size and validated by functional experiments.

\section{Supplementary information}

Supplementary information accompanies this paper at https://doi.org/10. 1186/s12883-019-1504-2.

\begin{abstract}
Additional file 1: Figure S1. Kaplan-Meier curves for overall survival and progression-free survival according to surgical method and use of chemotherapy in patients with glioma. A: Kaplan-Meier curves of overall survival in different surgical method; B: Kaplan-Meier curves of progressionfree survival in different surgical method; C: Kaplan-Meier curves of overall survival according to Chemotherapy or not; D: Kaplan-Meier curves of progression-free survival according to Chemotherapy or not.
\end{abstract}

Additional file 2: Figure S2. AKAP6 gene expression is up-regulated in glioma compared with that in normal tissues. Data was extracted from the GEPIA database (http://gepia.cancer-pku.cn/). The Y-axis represents the relative level of AKAP6 gene expression. GBM: glioblastoma multiforme. * indicates statistical significance $(p<0.01)$.

Additional file 3: Figure S3. Kaplan-Meier survival curves for overall survival based on AKAP6 gene in low-grade glioma. Data was extracted from the GEPIA database (http://gepia.cancer-pku.cn/).

Additional file 4: Table S1. The information and HWE about the candidate SNPS in AKAP6.

Additional file 5: Table S2. The impact of clinical factors on glioma patient OS and PFS.

\section{Abbreviations}

AKAP6: Kinase-anchored protein 6; Cl: Confidence intervals; GWAS: Genomewide association studies; HR: Hazard ratio; HWE: Hardy-Weinberg equilibrium; OR: Odds ratios; OS: Overall survival; PFS: Progression free survival; SNPs: Single-nucleotide polymorphisms; WHO: World Health Organization

\section{Acknowledgments}

We are grateful to the individuals who participated in this study. We also thank the clinicians and hospital staff who contributed to the sample and data collection for this study. 


\section{Authors' contributions}

Conceptualization, YW; methodology, MZ; software, YZ; data curation, MZ and $\mathrm{JZ}$; writing, review and editing, $\mathrm{MZ}$; visualization, $\mathrm{YZ}$ and $\mathrm{TH}$. All authors read and approved the final manuscript.

\section{Funding}

This work was supported by the Fundamental Research Funds for the Central Universities [grant number: xij2015018]; the special research fund for personnel training of the Second Affiliated Hospital of Xi'an Jiaotong University [grant number: RC (XM) 201603]; and a special research fund for the Youth Sciences Foundation of the Second Affiliated Hospital of Xi'an Jiaotong University [grant number: YJ (QN) 201402].

\section{Availability of data and materials}

The datasets used and analyzed in the current study are available from the corresponding author on reasonable request.

\section{Ethics approval and consent to participate}

This study was approved by the ethics committee of Second Affiliated Hospital of Xi'an Jiaotong University and followed the Helsinki declaration. Each subject was informed of the purpose of our study and signed a written informed consent.

\section{Consent for publication}

All the authors agreed to publish the manuscript.

\section{Competing interests}

The authors declare that they have no conflict of interest.

\section{Author details}

'Department of Neurosurgery, Second Affiliated Hospital of Xi'an Jiaotong University, Xi'an 710004, Shaanxi, China. ${ }^{2}$ Department of Oncology, Second Affiliated Hospital of Xi'an Jiaotong University, Xi'an 710004, Shaanxi, China. ${ }^{3}$ Department of Neurosurgery, First Affiliated Hospital of Xi'an Jiaotong University, Xi'an 710061, Shaanxi, China. ${ }^{4}$ Department of Critical Care Medicine, the Second Affiliated Hospital of Xi'an Jiaotong University, \#157 Xiwu Road, Xi'an 710004, Shaanxi, China.

Received: 18 June 2019 Accepted: 20 October 2019

Published online: 23 November 2019

\section{References}

1. Amirian ES, Armstrong GN, Zhou R, Lau CC, Claus EB, Barnholtz-Sloan JS, et al. The glioma international case-control study: a report from the genetic epidemiology of Glioma international consortium. Am J Epidemiol. 2015; 183(2):85-91.

2. Li G, Zhang Z, Zhang J, Jin T, Liang H, Gong L, et al. Occipital anaplastic oligodendroglioma with multiple organ metastases after a short clinical course: a case report and literature review. Diagn Pathol. 2014;9(1):17.

3. Lawson HC, Sampath P, Bohan E, Park MC, Hussain N, Olivi A, et al. Interstitial chemotherapy for malignant gliomas: the Johns Hopkins experience. J Neuro-Oncol. 2007:83(1):61-70.

4. Anjum K, Shagufta BI, Abbas SQ, Patel S, Khan I, Shah SAA, et al. Current status and future therapeutic perspectives of glioblastoma multiforme (GBM) therapy: a review. Biomed Pharmacother. 2017;92:681-9.

5. Jiang N, Peng Y, Wang X, Dou C, He W. Assessing the association between EFEMP1 rs3791679 polymorphism and risk of glioma in a Chinese Han population. Genet Mol Res. 2016:15(3):gmr.15038279.

6. Wang L, Li G, Liu N, Wang Z, Xu X, Qi J, et al. Association of genetic variants in the CART gene with glioma susceptibility in a Chinese population. Oncotarget. 2016;5:1-7.

7. bo Jin T, Du S, kai Zhu X, Li G, Ouyang Y, He N, et al. Polymorphism in the IL4R gene and clinical features are associated with glioma prognosis: Analyses of case-cohort studies. Medicine. 2016;95(31):e4231.

8. Li G, Jin T, Liang H, Zhang Z, He S, Tu Y, et al. RTEL1 tagging SNPs and haplotypes were associated with glioma development. Diagn Pathol. 2013; 8(1):83.

9. Mistry $\mathrm{AM}$, Vnencak-Jones $\mathrm{CL}$, Mobley $\mathrm{BC}$. Clinical prognostic value of the isocitrate dehydrogenase 1 single-nucleotide polymorphism rs11554137 in glioblastoma. J Neuro-Oncol. 2018;138(2):307-13.
10. Colledge M, Scott JD. AKAPs: from structure to function. Trends Cell Biol. 1999;9(6):216-21.

11. Chen TC, Hinton DR, Zidovetzki R, Hofman FM. Up-regulation of the CAMP/ PKA pathway inhibits proliferation, induces differentiation, and leads to apoptosis in malignant gliomas. Lab Investig. 1998;78(2):165-74.

12. Seshadri S, Fitzpatrick AL, Ikram MA, DeStefano AL, Gudnason V, Boada M, et al. Genome-wide analysis of genetic loci associated with Alzheimer disease. Jama. 2010;303(18):1832-40.

13. Wang K, Zhang H, Bloss C, Durvuri V, Kaye W, Schork N, et al. A genomewide association study on common SNPs and rare CNVs in anorexia nervosa. Mol Psychiatry. 2011;16(9):949.

14. Davies G, Marioni RE, Liewald DC, Hill WD, Hagenaars SP, Harris SE, et al Genome-wide association study of cognitive functions and educational attainment in UK biobank (N=112 151). Mol Psychiatry. 2016;21 (6):758.

15. Yuan $L$, Jin $T B$, Yin JK, Du XL, Wang $Q$, Dong $R$, et al. Polymorphisms of tumor-related genes IL-10, PSCA, MTRR and NOC3L are associated with the risk of gastric cancer in the Chinese Han population. Cancer Epidemiol. 2012;36(6):e366-e72

16. Chen Y, Wu Y, Huang X, Qu P, Li G, Jin T, et al. Leukocyte telomere length: a novel biomarker to predict the prognosis of glioma patients. J Cancer Res Clin Oncol. 2015;141(10):1739-47.

17. Jin T, Wang Y, Li G, Du S, Yang H, Geng T, et al. Analysis of difference of association between polymorphisms in the XRCC5, RPA3 and RTEL1 genes and glioma, astrocytoma and glioblastoma. Am J Cancer Res. 2015:5(7):2294.

18. Wesseling P, Capper D. WHO 2016 Classification of Gliomas. Neuropathol Appl Neurobiol. 2018:44(2):139-50.

\section{Publisher's Note}

Springer Nature remains neutral with regard to jurisdictional claims in published maps and institutional affiliations.

Ready to submit your research? Choose BMC and benefit from:

- fast, convenient online submission

- thorough peer review by experienced researchers in your field

- rapid publication on acceptance

- support for research data, including large and complex data types

- gold Open Access which fosters wider collaboration and increased citations

- maximum visibility for your research: over $100 \mathrm{M}$ website views per year

At BMC, research is always in progress.

Learn more biomedcentral.com/submissions 